\title{
Intrinsic origin of amyloid aggregation: Collective effects of the mutation and tautomerism of histidine
}

Hao Li $i^{\dagger,}$, Abbas Salimi ${ }^{\dagger, t}, \#$, and Jin Yong Lee ${ }^{\dagger, \xi^{*}}$

Department of Chemistry, Sungkyunkwan University, Suwon 440-746, Korea ${ }^{+}$School of Chemical Engineering, Sungkyunkwan University, Suwon 440-746, Korea

${ }^{\S}$ School of Chemistry and Chemical Engineering, University of Jinan, Jinan, 250022, China

${ }^{\#}$ These authors contributed equally to this work.

*Corresponding authors: jinylee@skku.edu(J. Y. Lee).

Figure S1. Cluster distributions (left) of each isomer, the most abundant structure (right).

Figure S2. The $\alpha$-helix contents of each amino acid of A2V A $\beta 40$ isomers.

Table S1. Hydrogen bond interactions of histidine residues of each isomer. 
$\varepsilon \varepsilon \varepsilon$

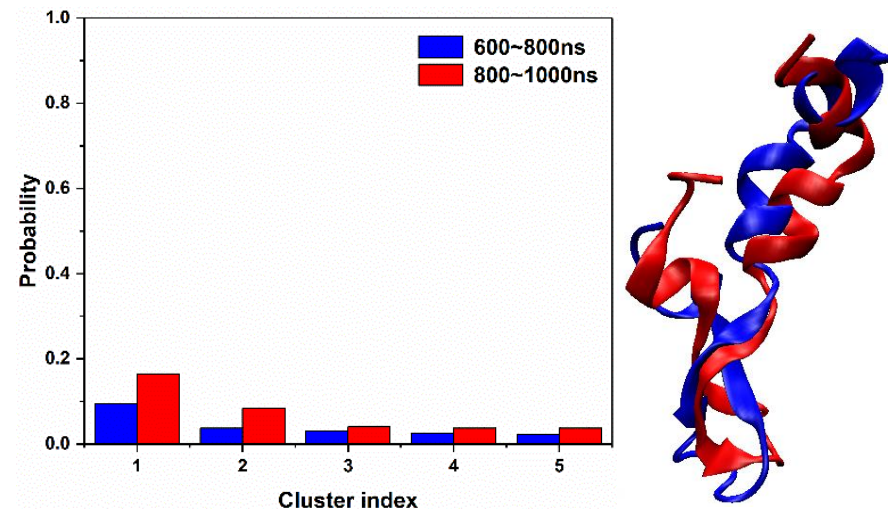

$\varepsilon \varepsilon \delta$

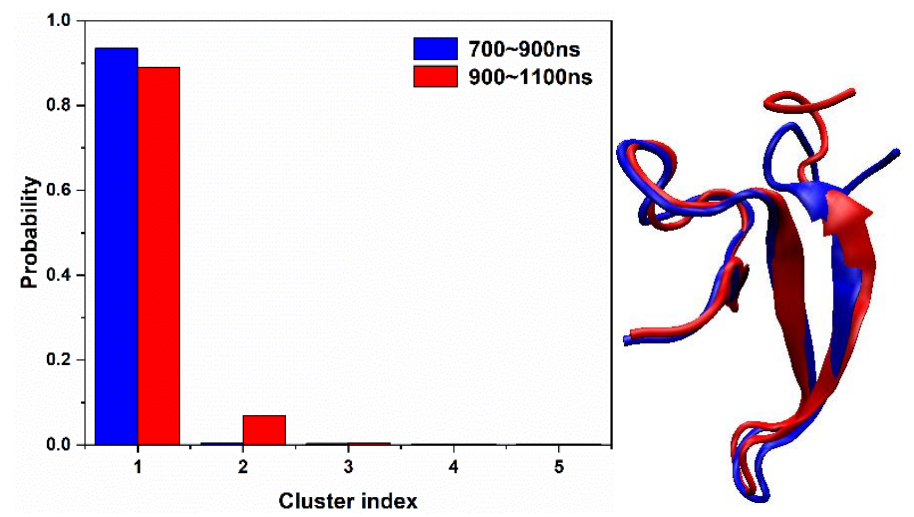

$\varepsilon \delta \varepsilon$
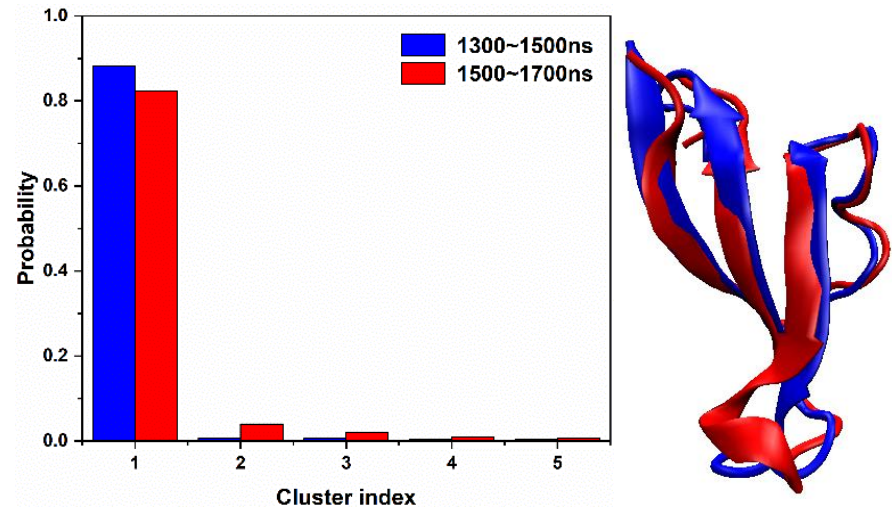

$\varepsilon \delta \delta$ 


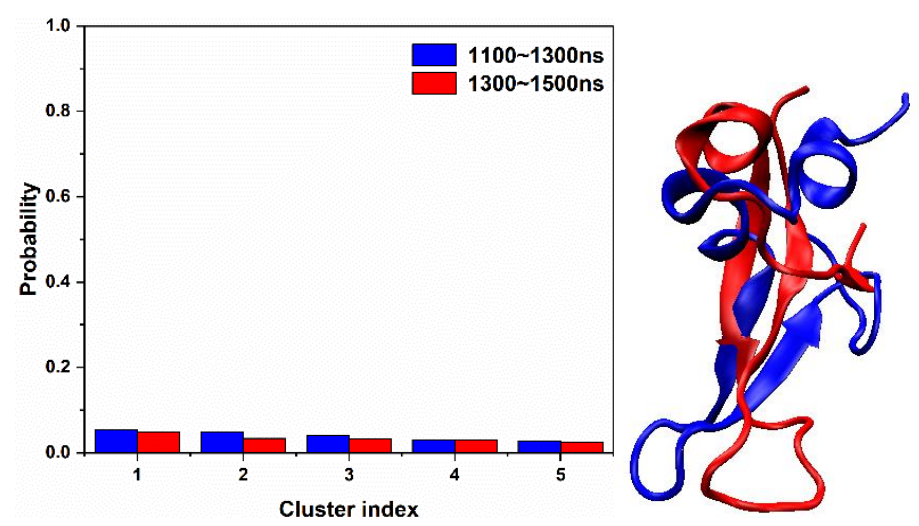

$\delta \varepsilon \varepsilon$
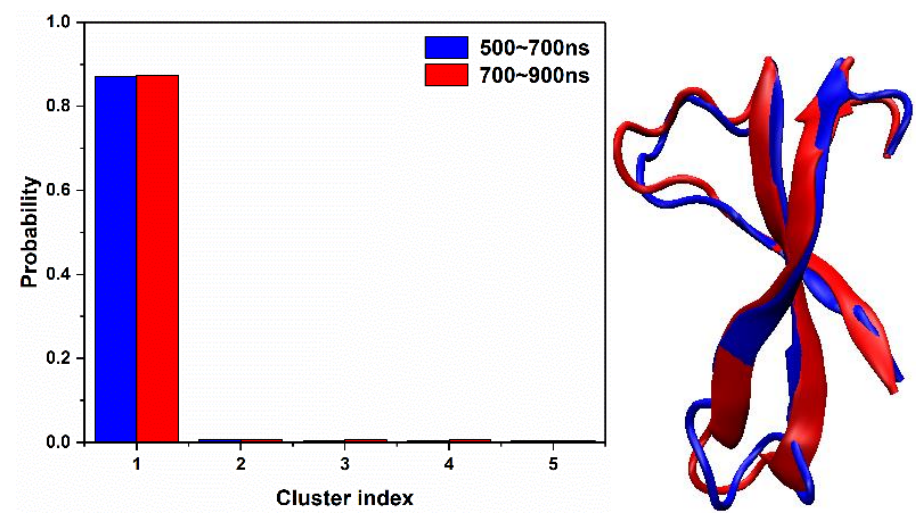

$\delta \varepsilon \delta$

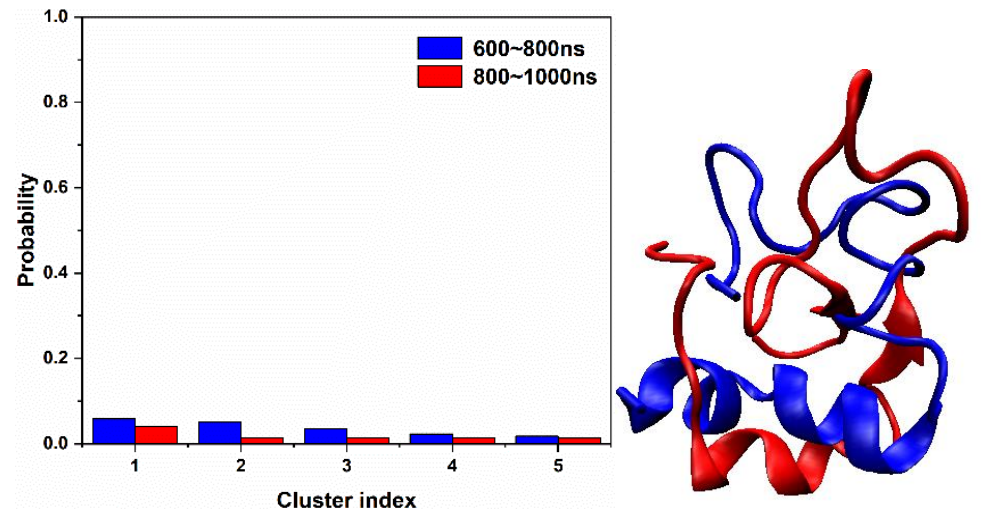

$\delta \delta \varepsilon$

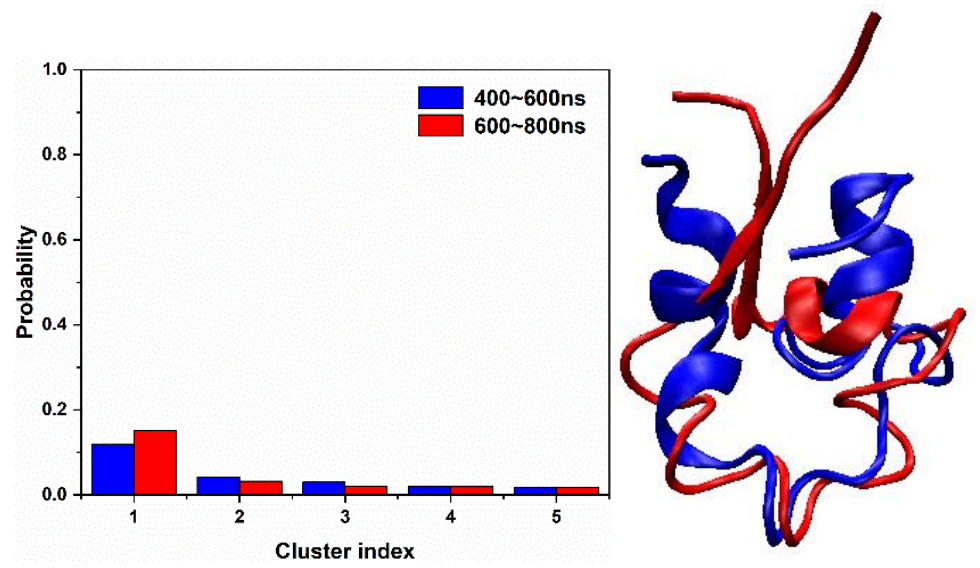


$\delta \delta \delta$

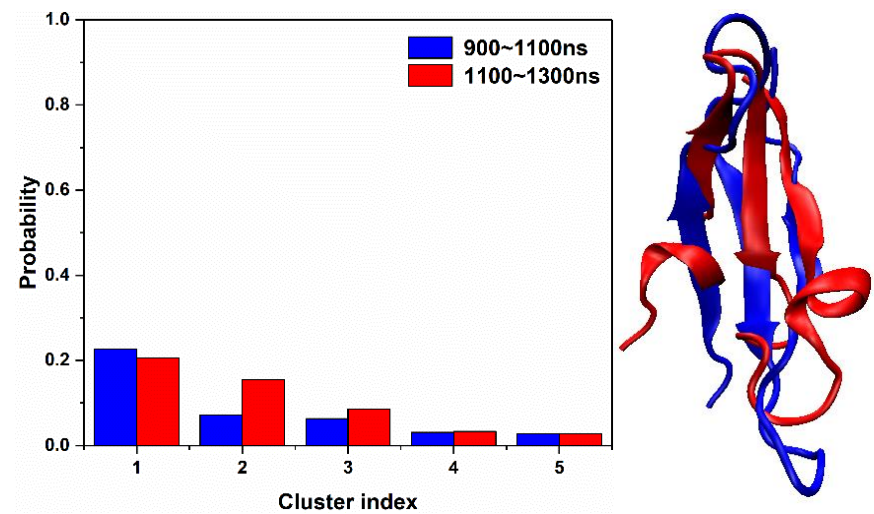

Figure S1. Cluster distributions (left) of each isomer, the most abundant structure (right).
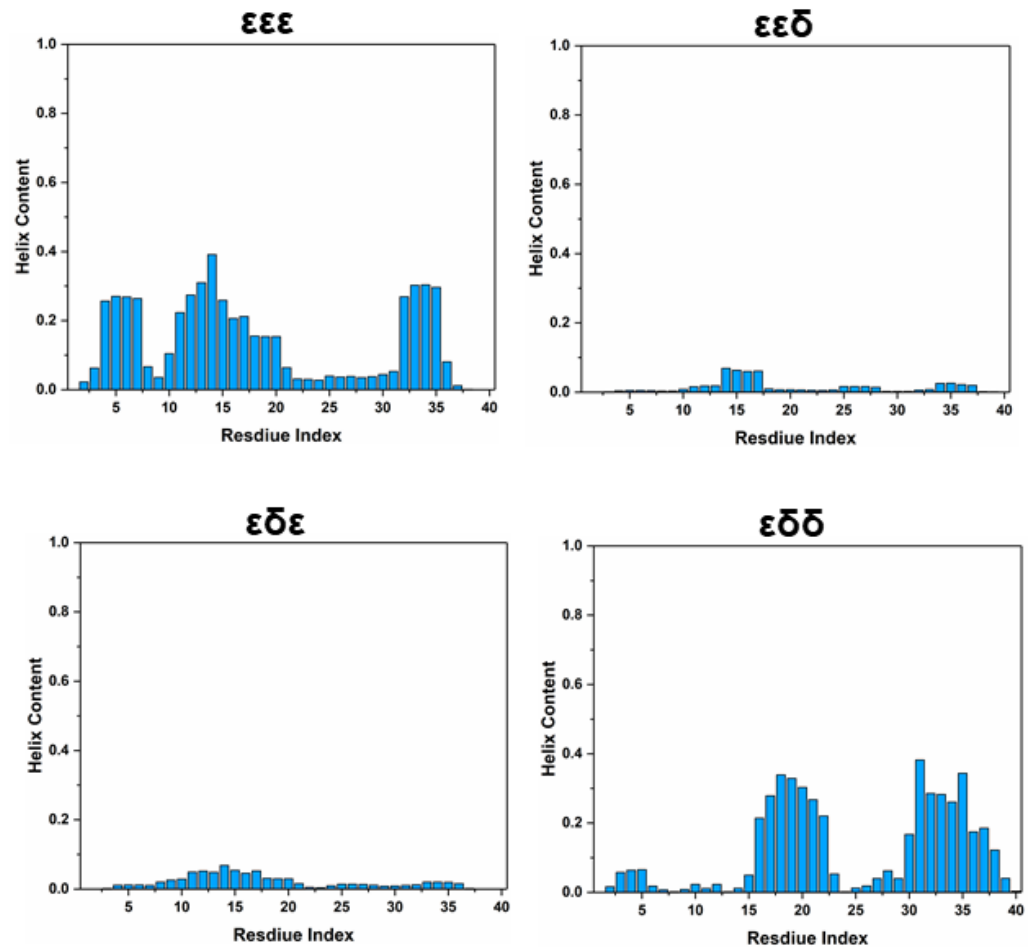

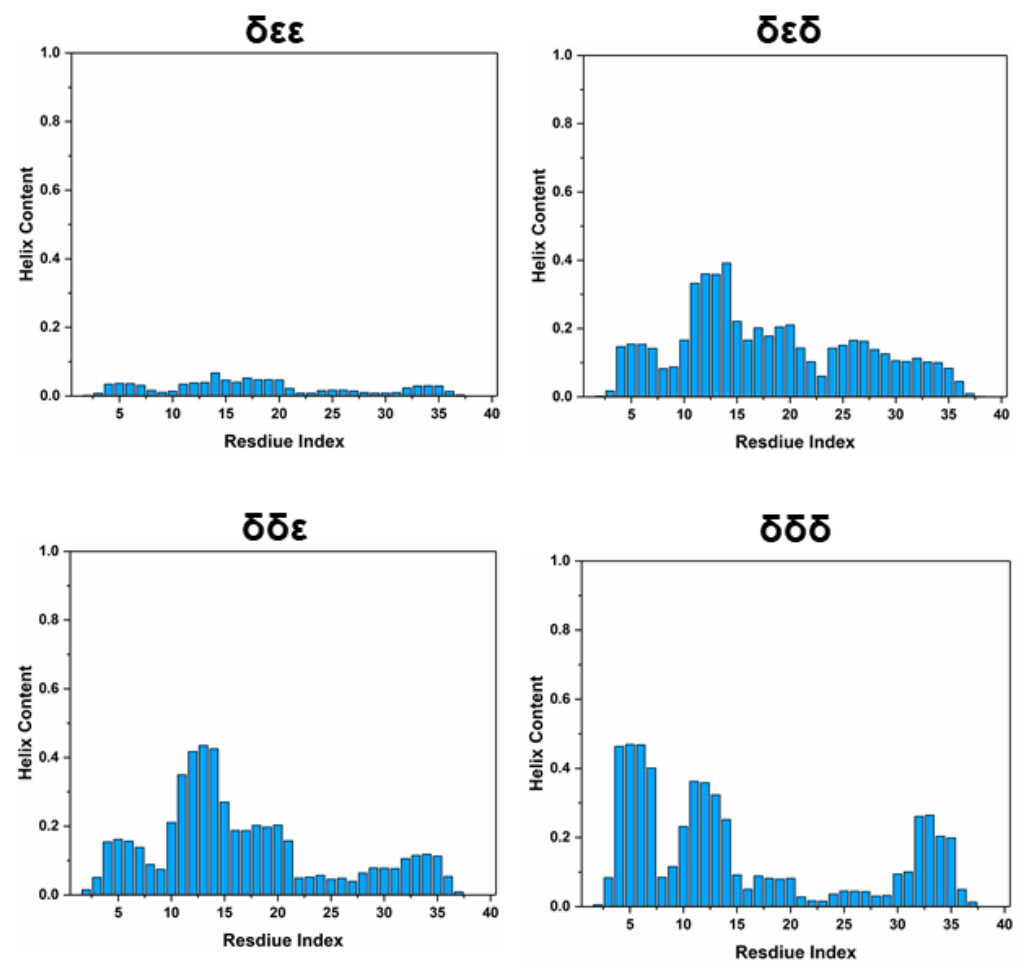

Figure S2. The $\alpha$-helix contents of each amino acid of A2V A $\beta 40$ isomers.

Table S1. Hydrogen bond interactions of histidine residues of each isomer.

\begin{tabular}{|c|c|c|c|c|c|c|}
\hline & \multicolumn{3}{|c|}{$\varepsilon \varepsilon \varepsilon$} & \multicolumn{3}{|c|}{$\varepsilon \varepsilon \delta$} \\
\hline & $\mathrm{P}$ & Acceptor & Donor & $\mathrm{P}$ & Acceptor & Donor \\
\hline \multirow[t]{2}{*}{ Donor His6 } & $9.14 \%$ & GLU_11@O & HIE_6@N & & & \\
\hline & $8.32 \%$ & GLU_3@O & HIE_6@N & & & \\
\hline Acceptor His6 & $9.99 \%$ & HIE_6@O & GLY_9@N & & & \\
\hline \multirow[t]{2}{*}{ Donor His13 } & $8.32 \%$ & & HIE_13@N & & & \\
\hline & $6.68 \%$ & & HIE_13@N & & & \\
\hline \multirow[t]{2}{*}{ Acceptor His13 } & $15.78 \%$ & HIE_13@O & LYS_16@N & & & \\
\hline & $13.53 \%$ & HIE_13@O & LEU_17@N & & & \\
\hline \multirow[t]{3}{*}{ Donor His 14} & $9.64 \%$ & TYR_10@O & HIE_14@N & $43.48 \%$ & GLU_11@O & HID_14@N \\
\hline & $5.25 \%$ & GLU_11@O & HIE_14@N & $18.14 \%$ & GLU_11@OE1 & HID_14@ND1 \\
\hline & & & & $17.91 \%$ & GLU_11@OE2 & HID_14@ND1 \\
\hline \multicolumn{7}{|l|}{ Acceptor His14 } \\
\hline & \multicolumn{3}{|c|}{$\varepsilon \delta \varepsilon$} & \multicolumn{3}{|c|}{$\varepsilon \delta \delta$} \\
\hline & $\mathrm{P}$ & Acceptor & Donor & $\mathrm{P}$ & Acceptor & Donor \\
\hline Donor His6 & & & & $5.49 \%$ & GLU_3@O & HIE_6@N \\
\hline
\end{tabular}




\begin{tabular}{|c|c|c|c|c|c|c|}
\hline \multicolumn{7}{|l|}{ Acceptor His6 } \\
\hline \multirow[t]{4}{*}{ Donor His13 } & $23.14 \%$ & TYR_10@O & HID_13@ND1 & $16.26 \%$ & GLY_9@O & HID_13@ND1 \\
\hline & $10.95 \%$ & HID_13@O & HID_13@ND1 & $8.86 \%$ & TYR_10@O & HID_13@N \\
\hline & & & & $8.09 \%$ & TYR_10@O & HID_13@ND1 \\
\hline & & & & $5.09 \%$ & GLU_11@OE1 & HID_13@ND1 \\
\hline \multirow[t]{2}{*}{ Acceptor His13 } & $47.43 \%$ & HID_13@O & GLN_15@N & & & \\
\hline & $10.95 \%$ & HID_13@O & HID_13@ND1 & & & \\
\hline \multirow[t]{4}{*}{ Donor His14 } & $39.49 \%$ & LEU_17@O & HIE_14@N & $13.20 \%$ & GLU_11@O & HID_14@N \\
\hline & & & & $11.15 \%$ & TYR_10@O & HID_14@N \\
\hline & & & & $10.31 \%$ & TYR_10@O & HID_14@ND1 \\
\hline & & & & $8.46 \%$ & GLU_11@O & HID_14@ND1 \\
\hline \multicolumn{7}{|l|}{ Acceptor His14 } \\
\hline & \multicolumn{3}{|c|}{$\delta \varepsilon \varepsilon$} & \multicolumn{3}{|c|}{$\delta \varepsilon \delta$} \\
\hline & $\mathrm{P}$ & Acceptor & Donor & $\mathrm{P}$ & Acceptor & Donor \\
\hline \multirow[t]{3}{*}{ Donor His6 } & $18.00 \%$ & ASP_7@O & HID_6@ND1 & $9.56 \%$ & GLU_3@O & HID_6@N \\
\hline & $5.63 \%$ & HID_6@O & HID_6@ND1 & $6.81 \%$ & GLU_11@OE1 & HID_6@ND1 \\
\hline & $5.48 \%$ & SER_8@O & HID_6@ND1 & $6.64 \%$ & GLU_11@OE2 & HID_6@ND1 \\
\hline Acceptor His6 & $5.63 \%$ & HID_6@O & HID_6@ND1 & & & \\
\hline \multirow[t]{2}{*}{ Donor His 13} & & & & $9.34 \%$ & TYR_10@O & HIE_13@N \\
\hline & & & & $5.09 \%$ & GLY_9@O & HIE_13@N \\
\hline \multirow[t]{2}{*}{ Acceptor His13 } & & & & $12.68 \%$ & HIE_13@O & LYS_16@N \\
\hline & & & & $8.99 \%$ & HIE_13@O & LEU_17@N \\
\hline \multirow[t]{4}{*}{ Donor His14 } & $32.77 \%$ & GLU_11@O & HIE_14@N & $12.31 \%$ & TYR_10@O & HID_14@HD1 \\
\hline & & & & $10.28 \%$ & GLU_11@O & HID_14@H \\
\hline & & & & $8.85 \%$ & TYR_10@O & HID_14@H \\
\hline & & & & $6.65 \%$ & GLU_11@O & HID_14@HD1 \\
\hline \multirow[t]{3}{*}{ Acceptor His14 } & $32.78 \%$ & HIE_14@O & GLY_37@N & & & \\
\hline & & $\delta \delta \varepsilon$ & & & $\delta \delta \delta$ & \\
\hline & $\mathrm{P}$ & Acceptor & Donor & $\mathrm{P}$ & Acceptor & Donor \\
\hline \multirow[t]{4}{*}{ Donor His6 } & $7.81 \%$ & ASP_7@O & HID_6@ND1 & $25.15 \%$ & GLU_3@O & HID_6@N \\
\hline & $6.90 \%$ & GLU_3@O & HID_6@N & $12.77 \%$ & PHE_20@O & HID_6@ND1 \\
\hline & $6.53 \%$ & GLU_11@OE1 & HID_6@ND1 & & & \\
\hline & $5.48 \%$ & GLU_11@OE2 & HID_6@ND1 & & & \\
\hline \multicolumn{7}{|l|}{ Acceptor His6 } \\
\hline \multirow[t]{5}{*}{ Donor His13 } & $15.83 \%$ & GLY_9@O & HID_13@ND1 & $14.76 \%$ & GLY_9@O & HID_13@ND1 \\
\hline & $11.35 \%$ & TYR_10@O & HID_13@N & $6.88 \%$ & TYR_10@O & HID_13@N \\
\hline & $7.80 \%$ & GLU_11@OE2 & HID_13@ND1 & & & \\
\hline & $7.48 \%$ & TYR_10@O & HID_13@ND1 & & & \\
\hline & $7.45 \%$ & GLU_11@OE1 & HID_13@ND1 & & & \\
\hline \multirow[t]{2}{*}{ Acceptor His13 } & $16.32 \%$ & HID_13@O & LEU_17@N & $6.15 \%$ & HID_13@O & LYS_16@N \\
\hline & $14.09 \%$ & HID_13@O & LYS_16@N & & & \\
\hline \multirow[t]{3}{*}{ Donor His14 } & $17.82 \%$ & TYR_10@O & HIE_14@N & $11.31 \%$ & GLU_11@OE1 & HID_14@ND1 \\
\hline & $8.10 \%$ & GLU_11@O & HIE_14@N & $10.60 \%$ & GLU_11@OE2 & HID_14@ND1 \\
\hline & & & & $10.56 \%$ & TYR_10@O & HID_14@N \\
\hline
\end{tabular}




\begin{tabular}{llll}
\hline & $10.19 \%$ & TYR_10@O & HID_14@ND1 \\
& $7.57 \%$ & GLU_11@O & HID_14@N \\
Acceptor His14 & & & \\
\hline
\end{tabular}

